\title{
Estudo de Convergência de Aproximações por Elementos Finitos Híbridos Estabilizados para o Problema de Stokes
}

\author{
Iury Igreja, Cristiane Faria, Abimael F. D. Loula \\ Laboratório Nacional de Computação Científica, LNCC/IMCT, \\ Av. Getúlio Vargas, 333, Quitandinha, Petrópolis/RJ - CEP: 25651-075 \\ E-mail: \{iuryhai, cofaria, aloc\}@lncc.br
}

Resumo: Apresentamos um estudo comparativo da convergência de aproximações obtidas com elementos triangulares e quadrilaterais associados a formulações variacionais híbridas do problema de Stokes em velocidade e pressão. São analisados os métodos de elementos finitos híbridos estabilizados $\boldsymbol{S H 2 M}$ e $\boldsymbol{S H 3 M}$ desenvolvidos em [10]. Os métodos em questão são caracterizados pela imposição da continuidade de forma fraca via multiplicadores de Lagrange associados aos campos de velocidade ou de velocidade e pressão. Tal metodologia preserva as principais características dos métodos de Galerkin descontínuo como hp-adaptatividade, porém com menor complexidade e custo computacional. Resultados numéricos mostram que as formulações em questão permitem o uso de espaços de elementos finitos que empregam aproximações polinomiais, lagrangianas ou não, de igual ordem para velocidade e pressão tanto para elementos triangulares quanto para elementos quadrilaterais. Como esperado, para igual número de graus de liberdade observamos uma melhor precisão dos elementos quadriláteros comparados com os elementos triangulares.

Palavras-chave: Métodos de Elementos Finitos, Métodos Híbridos, Métodos de Galerkin Descontínuo, Problema de Stokes, Estabilizações.

\section{Introdução}

Métodos de Galerkin Descontínuo (DG) apresentam grande flexibilidade na implementação de estratégias de adaptatividade do tipo $h p$, além de possibilitar o uso de espaços de elementos finitos que consistem em polinômios descontínuos por partes. Deste modo, vários autores têm se dedicado ao desenvolvimento de métodos mistos de Galerkin descontínuo para a aproximação numérica de problemas de escoamento incompressível. Particularmente para o problema de Stokes, podemos citar os trabalhos de Baker et al [2], Cockburn et al [6] e Schötzau et al [12]. O preço das vantagens oferecidas pelos métodos DG são uma maior complexidade de formulação, implementação computacional e elevado número de graus de liberdade. Hibridizações para os métodos DG têm sido propostas com o intuito de derivar novos métodos de elementos finitos com igual ou mesmo melhor estabilidade e reduzido custo computacional, preservando a robustez e a flexibilidade dos métodos DG [5, 8].

Métodos de elementos finitos híbridos surgiram a partir do trabalho de Veubeke [9] que foi compreendido como uma técnica de implementação associada a já conhecida manipulação algébrica matricial denominada condensação estática. Posteriormente, Arnold et al [1] mostraram que a hibridização é mais do que um truque de implementação, uma vez que ficou provado que a nova variável que impoem de forma fraca a continuidade do fluxo em cada aresta, interpretada como o multiplicador de Lagrange, contém informações adicionais sobre a solução exata $[11,3]$. Na última década, hibridizações de diversas classes de métodos de Galerkin descontínuo (HDG) foram introduzidas por Cockburn e seus colaboradores [4, 5, 7].

Neste contexto, propomos dois métodos de elementos finitos híbridos estabilizados de Galerkin descontínuo em [10] usando elementos triangulares para o problema de Stokes. E neste 
trabalho, apresentaremos novos resultados numéricos de estudos de convergência para as aproximações dos campos de velocidade e pressão utilizando elementos triangulares e quadrilaterais. Tais métodos permitem o emprego de interpolações de mesma ordem para a aproximação dos campos de velocidade e pressão. A primeira formulação identificada por SH3M envolve três multiplicadores de Lagrange que estão associados com as duas componentes da velocidade e com a pressão e pode ser visto como uma versão híbrida do método DG analisado por Schötzau et al [12]. A segunda formulação é uma versão hibridizada do método DG proposto por Schötzau et al [13] caracterizada pela introdução de multiplicadores apenas para o campo de velocidade (SH2M). Tal aproximação pode ser encontrada em Egger et al [8], mas diferentemente deste trabalho, aplicamos uma estratégia de implementação onde introduzimos, no nível de cada elemento, um termo de regularização que confere unicidade de solução para o campo de pressão.

Este trabalho é organizado como segue. Na Seção 2, apresentamos o problema modelo. Notações e definições são introduzidas na Seção 3. Os métodos SH3M e SH2M são recapitulados na Seção 4. A metodologia de resolução destes métodos são desenvolvidas na Seção 5. Na Seção 6, resultados comparando as soluções aproximadas para elementos triangulares e quadrilaterais são apresentados. Finalmente, na Seção 7, apresentamos as conclusões deste trabalho.

\section{Problema Modelo}

Seja $\Omega$ um domínio limitado em $\mathbb{R}^{2}$, com contorno $\Gamma=\partial \Omega$. O escoamento de um fluido viscoso incompressível confinado em $\Omega$ com condição de contorno de Dirichlet homogênea é modelado pelo seguinte sistema de equações diferenciais parciais, conhecido como problema de Stokes

Dada a função $\mathbf{f}: \Omega \rightarrow \mathbb{R}^{2}$, encontrar $\mathbf{u}: \Omega \rightarrow \mathbb{R}^{2}$ e $p: \Omega \rightarrow \mathbb{R}$, tal que

$$
\begin{array}{rrr}
-\nu \Delta \mathbf{u}+\nabla p=\mathbf{f} & \text { em } & \Omega, \\
\operatorname{div} \mathbf{u}=0 & \text { em } & \Omega, \\
\mathbf{u}=\mathbf{g}_{D}=\mathbf{0} & \text { sobre } & \Gamma,
\end{array}
$$

onde $\nu>0$ denota a viscosidade, $\Delta$ o operador Laplaciano, u o campo de velocidade e $p$ a pressão hidrostática. A equação (3) satisfaz a condição usual de compatibilidade $\int_{\Omega} \mathbf{g}_{D} \cdot \mathbf{n} d \mathbf{x}=0$, com $\mathbf{n}$ denotando o vetor unitário normal exterior a $\Gamma$.

\section{Notações e Definições}

Inicialmente, recordamos algumas notações e definições necessárias para a introdução das formulações híbridas estabilizadas para o nosso problema modelo. Dessa forma, seja $L^{2}(\Omega)$ o espaço das funções quadrado integráveis equipado com o produto interno e a norma usual e $L_{0}^{2}(\Omega)=\left\{q \in L^{2}(\Omega): \int_{\Omega} q d \mathbf{x}=0\right\}$. Restrigindo ao caso em duas dimensões $(d=2)$, denotamos uma união de todos os elementos $K, \mathcal{T}_{h}$, como uma partição regular de elementos finitos do domínio $\Omega$. Além disso, $\mathcal{E}_{h}$ denota o conjunto de todas as arestas $e$ de todos os elementos $K$ da malha $\mathcal{T}_{h}, \mathcal{E}_{h}^{0}$ o conjunto das arestas interiores e $\mathcal{E}_{h}^{\partial}=\mathcal{E}_{h} \cap \partial \Omega$ o conjunto de todas as arestas $\mathcal{E}_{h}$ sobre o contorno de $\Omega$. Para cada aresta $e$ associamos um vetor unitário normal $\mathbf{n}_{e}$.

Seja $\mathbf{V}_{h}$ e $Q_{h}$ os espaços das funções quebradas na partição de $\mathcal{T}_{h}$, dados por

$$
\begin{gathered}
\mathbf{V}_{h}^{k}=\left\{\mathbf{v} \in\left[L^{2}(\Omega)\right]^{2} ;\left.\mathbf{v}_{h}\right|_{K} \in\left[S_{k}(K)\right]^{2}, \forall K \in \mathcal{T}_{h}\right\} \\
Q_{h}^{l}=\left\{q \in\left(L^{2}(\Omega)\right) ;\left.q_{h}\right|_{K} \in S_{l}(K), \forall K \in \mathcal{T}_{h}\right\}
\end{gathered}
$$

onde $S_{k}(K)=P_{k}(K)$, denota o espaço de funções polinomiais de grau $k$ associado com elementos triangulares e $S_{k}(K)=Q_{k}(K)$, o espaço de funções polinomiais de grau $k$ associado a elementos quadrilaterais. Para introduzir os métodos híbridos, definimos os seguintes espaços associados aos multiplicadores de Lagrange

$$
\mathcal{M}_{h}^{m}=\left\{\boldsymbol{\mu}_{\boldsymbol{u}} \in\left[L^{2}\left(\mathcal{E}_{h}\right)\right]^{2}:\left.\boldsymbol{\mu}_{\boldsymbol{u}}\right|_{e}=\left[p_{m}(e)\right]^{2}, \forall e \in \mathcal{E}_{h}^{0},\left.\boldsymbol{\mu}_{\boldsymbol{u}}\right|_{e}=\mathbf{0}, \forall e \in \mathcal{E}_{h}^{\partial}\right\}
$$




$$
\mathcal{W}_{h}^{m}=\left\{\mu_{p} \in L^{2}\left(\mathcal{E}_{h}\right):\left.\mu_{p}\right|_{e}=p_{m}(e), \forall e \in \mathcal{E}_{h}^{0}\right\} .
$$

Similarmente, $p_{m}(e)$ expressa o espaço das funções polinomiais de grau $m$ sobre cada aresta $e$.

\section{Formulações Híbridas DG Estabilizadas}

Nesta seção, apresentamos duas formulações híbridas estabilizadas de Galerkin descontínuo para o problema de Stokes (1). A primeira, denominada SH3M, emprega os multiplicadores $\boldsymbol{\lambda}_{\boldsymbol{u}}$ e $\lambda_{p}$ definidos como o traço de $\mathbf{u}, \boldsymbol{\lambda}_{\boldsymbol{u}}=\left.\mathbf{u}\right|_{e}$ e o traço de $p, \lambda_{p}=\left.p\right|_{e}$ sobre cada aresta $e \in \mathcal{E}_{h}$, consiste em:

Encontrar $\left[\mathbf{u}_{h}, p_{h}\right] \in \mathbf{V}_{h}^{k} \times Q_{h}^{l}$ e os multiplicadores de Lagrange $\left[\boldsymbol{\lambda}_{\boldsymbol{u}_{\boldsymbol{h}}}, \lambda_{p_{h}}\right] \in \mathcal{M}_{h}^{m} \times \mathcal{W}_{h}^{m}$, satisfazendo

$$
\begin{aligned}
& \sum_{K \in \mathcal{T}_{h}} \int_{K} \nu \nabla \mathbf{u}_{h}: \nabla \mathbf{v}_{h} d \mathbf{x}-\sum_{K \in \mathcal{T}_{h}} \int_{K} p_{h} \operatorname{div} \mathbf{v}_{h} d \mathbf{x}-\sum_{K \in \mathcal{T}_{h}} \int_{K} \operatorname{div} \mathbf{u}_{h} q_{h} d \mathbf{x} \\
& +\sum_{K \in \mathcal{T}_{h}} \int_{\partial K} \lambda_{p_{h}}\left(\mathbf{v}_{h} \cdot \mathbf{n}_{K}\right) d s+\sum_{K \in \mathcal{T}_{h}} \int_{\partial K} \mu_{p_{h}}\left(\mathbf{u}_{h} \cdot \mathbf{n}_{K}\right) d s \\
& -\sum_{K \in \mathcal{T}_{h}} \int_{\partial K}\left(\nu \nabla \mathbf{u}_{h} \mathbf{n}_{K}\right) \cdot\left(\mathbf{v}_{h}-\boldsymbol{\mu}_{\boldsymbol{u}_{\boldsymbol{h}}}\right) d s-\sum_{K \in \mathcal{T}_{h}} \int_{\partial K}\left(\nu \nabla \mathbf{v}_{h} \mathbf{n}_{K}\right) \cdot\left(\mathbf{u}_{h}-\boldsymbol{\lambda}_{\boldsymbol{u}_{\boldsymbol{h}}}\right) d s \\
& +\beta_{u} \sum_{K \in \mathcal{T}_{h}} \int_{\partial K}\left(\mathbf{u}_{h}-\boldsymbol{\lambda}_{\boldsymbol{u}_{\boldsymbol{h}}}\right) \cdot\left(\mathbf{v}_{h}-\boldsymbol{\mu}_{\boldsymbol{u}_{\boldsymbol{h}}}\right) d s+\beta_{p} \sum_{K \in \mathcal{T}_{h}} \int_{\partial K}\left(p_{h}-\lambda_{p_{h}}\right)\left(q_{h}-\mu_{p_{h}}\right) d s \\
& =\sum_{K \in \mathcal{T}_{h}} \int_{K} \mathbf{f} \cdot \mathbf{v}_{h} d \mathbf{x}, \quad \forall\left[\mathbf{v}_{h}, q_{h}\right] \in \mathbf{V}_{h}^{k} \times Q_{h}^{l} \quad \mathrm{e} \quad \forall\left[\boldsymbol{\mu}_{\boldsymbol{u}_{h}}, \mu_{p_{h}}\right] \in \mathcal{M}_{h}^{m} \times \mathcal{W}_{h}^{m} .
\end{aligned}
$$

Os parâmetros de estabilização $\beta_{u}$ e $\beta_{p}$ são definidos como:

$$
\beta_{u}=\frac{\nu \beta_{0}}{h} \quad \text { e } \quad \beta_{p}=\frac{h \beta_{0}}{\nu}, \quad \text { com } \quad \beta_{0}>0 .
$$

A segunda formulação (SH2M) desenvolvida em [10] apresenta multiplicadores de Lagrange apenas para as componentes da velocidade definidos como o traço de $\mathbf{u}, \boldsymbol{\lambda}_{\boldsymbol{u}}=\left.\mathbf{u}\right|_{e}$ sobre cada aresta $e \in \mathcal{E}_{h}$, e pode ser formulada como

Encontrar o par $\left[\mathbf{u}_{h}, p_{h}\right] \in \mathbf{V}_{h}^{k} \times Q_{h}^{l}$ e o multiplicador de Lagrange $\boldsymbol{\lambda}_{\boldsymbol{u}_{\boldsymbol{h}}} \in \mathcal{M}_{h}^{m}$ tal que, para todo $\left[\mathbf{v}_{h}, q_{h}, \boldsymbol{\mu}_{\boldsymbol{u}_{\boldsymbol{h}}}\right] \in \mathbf{V}_{h}^{k} \times Q_{h}^{l} \times \mathcal{M}_{h}^{m}$

$$
\begin{aligned}
& \sum_{K \in \mathcal{T}_{h}} \int_{K} \nu \nabla \mathbf{u}_{h}: \nabla \mathbf{v}_{h} d \mathbf{x}-\sum_{K \in \mathcal{T}_{h}} \int_{K} p_{h} \operatorname{div} \mathbf{v}_{h} d \mathbf{x}-\sum_{K \in \mathcal{T}_{h}} \int_{K} \operatorname{div} \mathbf{u}_{h} q_{h} d \mathbf{x} \\
+ & \sum_{K \in \mathcal{T}_{h}} \int_{\partial K} p_{h} \mathbf{n}_{K} \cdot\left(\mathbf{v}_{h}-\boldsymbol{\mu}_{\boldsymbol{u}_{\boldsymbol{h}}}\right) d s+\sum_{K \in \mathcal{T}_{h}} \int_{\partial K} q_{h} \mathbf{n}_{K} \cdot\left(\mathbf{u}_{h}-\boldsymbol{\lambda}_{\boldsymbol{u}_{\boldsymbol{h}}}\right) d s \\
- & \sum_{K \in \mathcal{T}_{h}} \int_{\partial K}\left(\nu \nabla \mathbf{u}_{h} \mathbf{n}_{K}\right) \cdot\left(\mathbf{v}_{h}-\boldsymbol{\mu}_{\boldsymbol{u}_{\boldsymbol{h}}}\right) d s-\sum_{K \in \mathcal{T}_{h}} \int_{\partial K}\left(\nu \nabla \mathbf{v}_{h} \mathbf{n}_{K}\right) \cdot\left(\mathbf{u}_{h}-\boldsymbol{\lambda}_{\boldsymbol{u}_{\boldsymbol{h}}}\right) d s \\
+ & \beta_{u} \sum_{K \in \mathcal{T}_{h}} \int_{\partial K}\left(\mathbf{u}_{h}-\boldsymbol{\lambda}_{\boldsymbol{u}_{\boldsymbol{h}}}\right) \cdot\left(\mathbf{v}_{h}-\boldsymbol{\mu}_{\boldsymbol{u}_{\boldsymbol{h}}}\right) d s=\sum_{K \in \mathcal{T}_{h}} \int_{K} \mathbf{f} \cdot \mathbf{v}_{h} d \mathbf{x}
\end{aligned}
$$

O parâmetro $\beta_{u}$ adotado aqui é o mesmo definido em (9).

\section{Metodologia de Resolução}

Como estratégia de resolução objetivamos a eliminação dos graus de liberdade das variáveis

$\mathbf{u}_{h}$ e $p_{h}$ no nível do elemento em favor dos graus de liberdade do multiplicador, gerando um 
sistema global em função apenas dos multiplicadores de Lagrange. Dessa forma, primeiramente adicionamos um termo de regularização do tipo $\frac{\varepsilon}{\nu} \int_{K} p_{h} q_{h} d \mathbf{x}$ com o intuito de assegurar unicidade de solução do campo de pressão. Em seguida, dividimos as formulações (8) e (10) em um problema local definido em cada elemento para as variáveis velocidade e pressão acoplado a um problema global para o multiplicador. Esta idéia pode ser escrita da seguinte forma matricial

$$
\begin{aligned}
\mathbf{A}_{K} \mathbf{U}+\mathbf{B}_{K} \boldsymbol{\Lambda} & =\mathbf{F}_{K}, \quad \forall K \in \mathcal{T}_{h} \\
\sum_{K \in \mathcal{T}_{h}} \mathbf{B}_{K}^{T} \mathbf{U}+\sum_{K \in \mathcal{T}_{h}} \mathbf{C}_{K} \boldsymbol{\Lambda} & =\mathbf{0},
\end{aligned}
$$

onde $\mathbf{U}=\{\mathbf{u}, p\}$ e $\boldsymbol{\Lambda}=\left\{\boldsymbol{\lambda}_{\boldsymbol{u}_{\boldsymbol{h}}}, \lambda_{p_{h}}\right\}$ para o método SH3M e $\boldsymbol{\Lambda}=\left\{\boldsymbol{\lambda}_{\boldsymbol{u}_{\boldsymbol{h}}}\right\}$ para SH2M. Dado que $\mathbf{A}_{K}$ é positiva definida, manipulamos o sistema (11) para obter

$$
\mathbf{U}=\mathbf{A}_{K}^{-1}\left(\mathbf{F}_{K}-\mathbf{B}_{K} \mathbf{\Lambda}\right), \quad \forall K \in \mathcal{T}_{h}
$$

Substituindo (13) em (12), obtemos o sistema global em função apenas do multiplicador como segue

$$
\sum_{K \in \mathcal{T}_{h}}\left(\mathbf{C}_{K}-\mathbf{B}_{K}^{T} \mathbf{A}_{K}^{-1} \mathbf{B}_{K}\right) \boldsymbol{\Lambda}=-\sum_{K \in \mathcal{T}_{h}} \mathbf{B}_{K}^{T} \mathbf{A}_{K}^{-1} \mathbf{F}_{K}
$$

Após resolver o sistema global (14), o vetor $\mathbf{U}$ é obtido a partir de (13) com $\boldsymbol{\Lambda}$ dado por (14).

\section{Resultados Numéricos}

Nesta seção, desenvolvemos experimentos numéricos computacionais para as formulações SH3M e SH2M. Os desempenhos destes métodos são testadas através de estudos de convergência realizados em um domínio quadrado $\Omega=(0,1) \times(0,1)$ com condição de contorno homogênea, onde a solução exata é dada por

$$
\mathbf{u}=\left[\begin{array}{c}
\sin (\pi x) \cos (\pi y) \\
-\cos (\pi x) \sin (\pi y)
\end{array}\right], \quad p=-2 \pi \cos (\pi x) \cos (\pi y),
$$

desde que

$$
\mathbf{f}=\left[\begin{array}{l}
2 \pi^{2}(1+\nu) \sin (\pi x) \cos (\pi y) \\
2 \pi^{2}(1-\nu) \cos (\pi x) \sin (\pi y)
\end{array}\right]
$$

A solução aproximada é obtida utilizando elementos triangulares $P_{k} P_{l}-p_{m}$ e quadrilaterais $Q_{k} Q_{l}-p_{m}$, onde $k, l$ e $m$ denotam o grau do espaço polinomial lagrangiano para a velocidade $\left(\mathbf{u}_{h}\right)$, a pressão $\left(p_{h}\right)$ e ambos os multiplicadores $\left(\boldsymbol{\lambda}_{\boldsymbol{u}_{\boldsymbol{h}}}, \lambda_{p_{h}}\right)$, respectivamente. Para os parâmetros $\varepsilon$ e $\nu$ adotamos $\varepsilon=10^{-5}$ e $\nu=2.0$ em todas as simulações.

A escolha dos parâmetros de estabilização definidos em (9) é feita com base na influência do $\beta_{0}$ na precisão da aproximação da velocidade. A Figura 1 apresenta resultados dos estudos realizados para identificarmos as melhores escolhas para o parâmetro $\beta_{0}$ para elementos triangulares $P 1 P 1-p 1$ e $P 2 P 1-p 2$ com malha fixa de 32 elementos e quadrilaterais $Q 1 Q 1-p 1$ e $Q 2 Q 1-p 1$ com malha fixa de 16 elementos. Estes estudos foram realizados em malhas fixas e pouco refinadas com o intuito de tornar as simulações menos custosas computacionalmente uma vez que o emprego de malhas mais refinadas não causam mudanças significativas no resultado. Dessa forma, para elementos triangulares, com o método SH3M os resultados apontam escolhas ótimas para $\beta_{0}=11$ quando $k=l=m=1$ e $\beta_{0}=22$ quando $l=1, k=m=2$ e com o método SH2M, $\beta_{0}=8$ quando $k=l=m=1$ e $\beta_{0}=19$ quando $l=1, k=m=2$. Por outro lado, para quadriláteros, temos $\beta_{0}=12$ para $k=l=m=1$ e $\beta_{0}=35$ para $l=1, k=m=2$ para o método SH3M e $\beta_{0}=11$ para $k=l=m=1$ e $\beta_{0}=33$ para $l=1, k=m=2$ para o método SH2M. Apesar de haver algum padrão entre os valores obtidos para $\beta_{0}$ não podemos escrever 
uma fórmula geral pois este padrão não se confirma para interpolações de mais alta ordem. Todavia, Schötzau et al [12] sugerem uma forma geral para os parâmetros $\beta_{u}$ e $\beta_{p}$, no entanto os valores obtidos utilizando esta forma em nossas formulações apresentam precisão inferior se comparado aos nossos resultados.

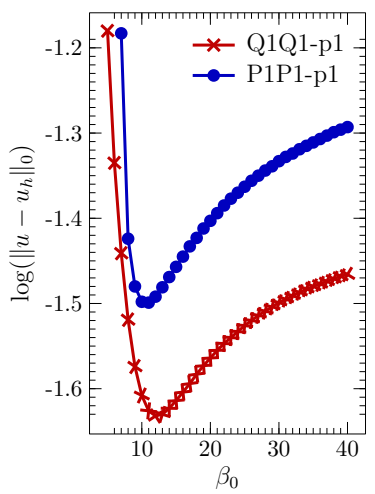

(a) SH3M

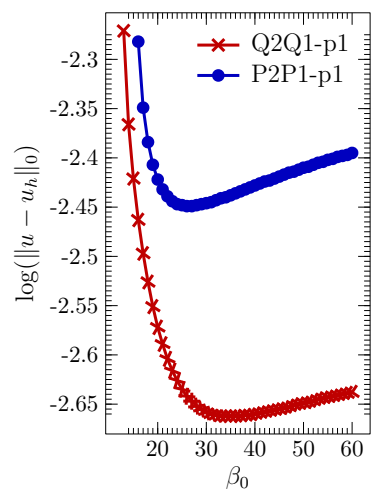

(b) SH3M

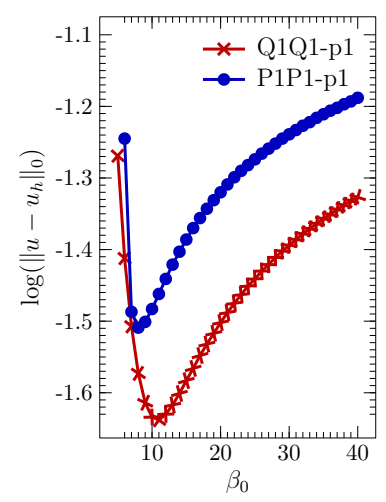

(c) $\mathrm{SH} 2 \mathrm{M}$

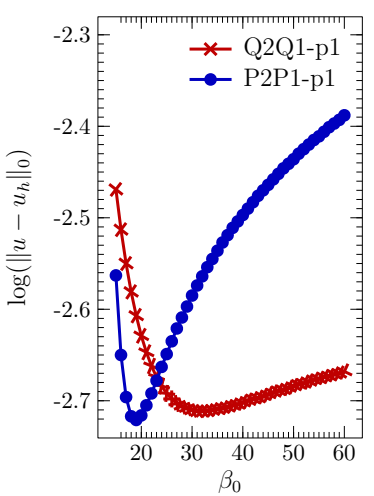

(d) SH2M

Figura 1: Influência do parâmetro $\beta_{0}$ na precisão da aproximação do método SH3M e SH2M.

Um estudo comparativo das taxas de convergência na norma $L^{2}(\Omega)$ obtidas com os métodos SH3M e SH2M para elementos triangulares e quadrilaterais para as variáveis $\mathbf{u}, p, \boldsymbol{\lambda}_{\boldsymbol{u}}$ e $\lambda_{p}$ é apresentado nas Figuras 2-5. Nestes experimentos usamos elementos triangulares $P_{1} P_{1}-p_{1}$, $P_{2} P_{1}-p_{2}$ e elementos quadrilaterais $Q_{1} Q_{1}-p_{1}, Q_{2} Q_{1}-p_{2}$. Para o método SH3M, nas Figuras 2(a)-2(d), utilizamos interpolações lineares para todas as variáveis. Observamos taxas ótimas de convergência para as aproximações para o campo de velocidade e multiplicadores e uma superconvergência para o campo de pressão para ambos elementos triangulares e quadrilaterais. Além disso, podemos notar que para todas as simulações os elementos quadrilaterais aproximam de forma mais precisa que os elementos triangulares.

Resultados numéricos que empregam aproximações polinomiais lagrangianas de diferente ordem para os campos de velocidade e pressão podem ser vistos nas Figuras 3(a)-3(d). Como no caso anterior, taxas ótimas de convergência são obtidas para as aproximações $\mathbf{u}_{h}, \boldsymbol{\lambda}_{\boldsymbol{u}_{\boldsymbol{h}}}$ e $\lambda_{p_{h}}$, além de uma superconvergência para a pressão. Observamos também, que para este caso o uso de elementos quadrilaterais se mostra mais preciso do que elementos triangulares.

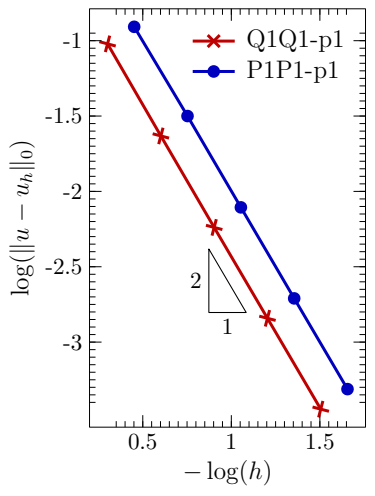

(a) $\mathbf{u}_{h}$

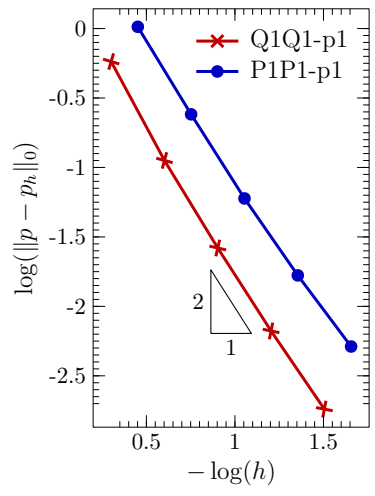

(b) $p_{h}$

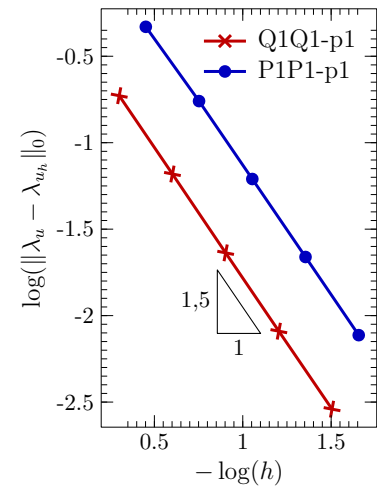

(c) $\boldsymbol{\lambda}_{u_{h}}$

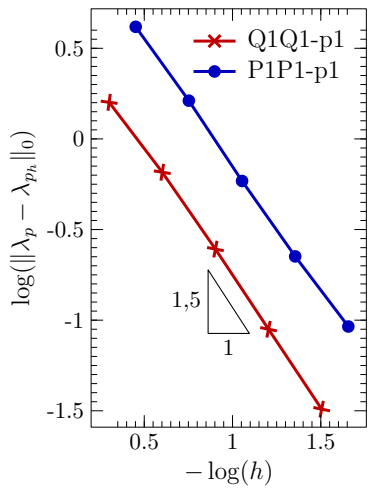

(d) $\lambda_{p_{h}}$

Figura 2: Estudo de convergência $h$ das aproximações $\mathbf{u}_{h}, p_{h}, \boldsymbol{\lambda}_{\boldsymbol{u}_{\boldsymbol{h}}}$ e $\lambda_{p_{h}}$ do método SH3M na norma $L^{2}(\Omega)$ comparando a precisão de elementos triangulares com elementos quadrilaterais utilizando interpolações lineares para ambos os campos e multiplicadores.

Para o método SH2M, os resultados apresentados nas Figuras 4(a)-4(c) confirmam taxa ótima de convergência para as aproximações $\mathbf{u}_{h}, p_{h}$ e $\boldsymbol{\lambda}_{\boldsymbol{u}_{\boldsymbol{h}}}$ tanto para elementos quadrilaterais quanto para triângulos para interpolações de mesma ordem para velocidade e pressão. Como no 
Proceeding Series of the Brazilian Society of Applied and Computational Mathematics, Vol. 3, N. 1, 2015.

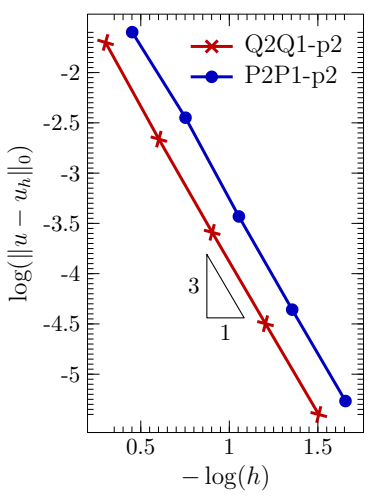

(a) $\mathbf{u}_{h}$

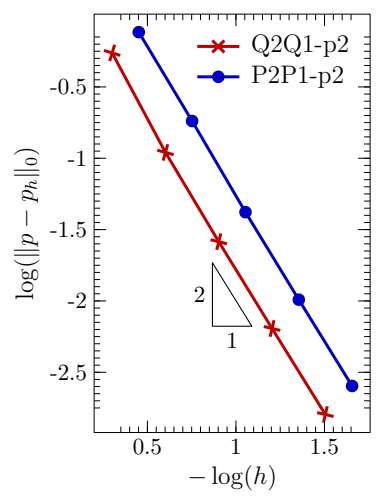

(b) $p_{h}$

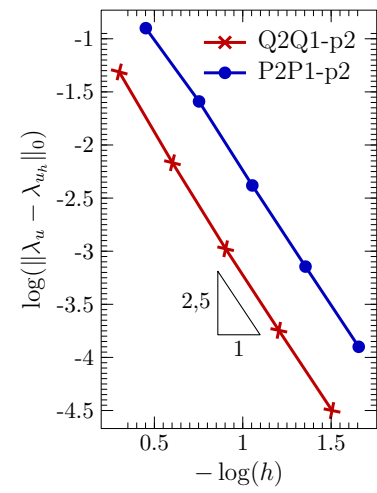

(c) $\lambda_{u_{h}}$

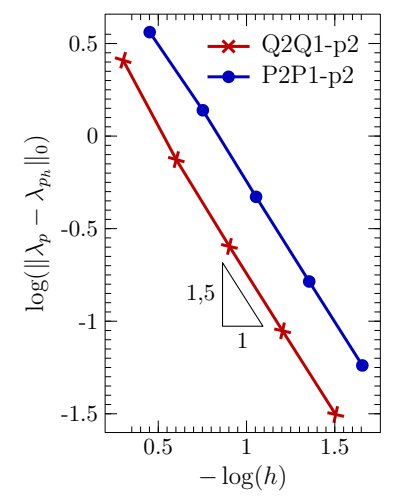

(d) $\lambda_{p_{h}}$

Figura 3: Estudo de convergência $h$ das aproximações $\mathbf{u}_{h}, p_{h}, \boldsymbol{\lambda}_{\boldsymbol{u}_{\boldsymbol{h}}}$ e $\lambda_{p_{h}}$ do método SH3M na norma $L^{2}(\Omega)$ comparando a precisão de elementos triangulares com elementos quadrilaterais utilizando interpolações quadráticas para a velocidade e multiplicadores e linear para a pressão.

método SH3M, percebemos que elementos quadrilaterais são mais precisos que os triangulares para as aproximações $\mathbf{u}_{h}$ e $\boldsymbol{\lambda}_{\boldsymbol{u}_{\boldsymbol{h}}}$, porém isto não se confirma para a pressão. As Figuras 5(a)-5(c) apresentam interpolações para velocidade e o multiplicador com uma ordem a mais que o campo de pressão, neste caso também podemos observar taxa ótima para as aproximações e uma maior precisão para elementos quadrilaterais para $p_{h}$ e $\boldsymbol{\lambda}_{\boldsymbol{u}_{h}}$.

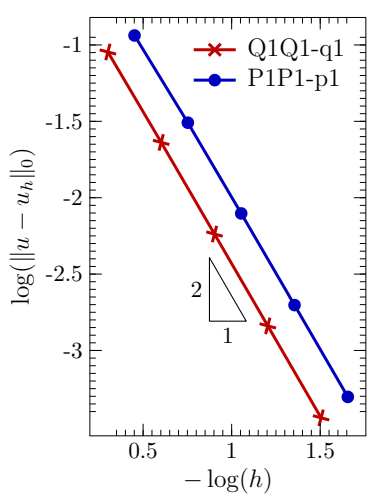

(a) $\mathbf{u}_{h}$

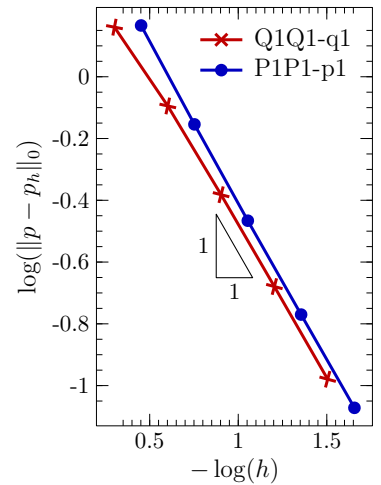

(b) $p_{h}$

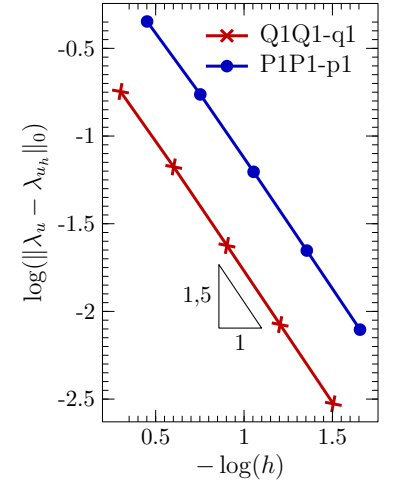

(c) $\boldsymbol{\lambda}_{u_{h}}$

Figura 4: Estudo de convergência $h$ das aproximações $\mathbf{u}_{h}, p_{h}$ e $\boldsymbol{\lambda}_{\boldsymbol{u}_{\boldsymbol{h}}}$ do método SH2M na norma $L^{2}(\Omega)$ comparando a precisão de elementos triangulares com elementos quadrilaterais utilizando interpolações lineares para ambos os campos e multiplicador.

\section{Conclusão}

Neste trabalho, apresentamos novos resultados numéricos utilizando elementos quadrilaterais para as formulações híbridas estabilizadas de Galerkin descontínuo propostas em [10]. Os métodos SH3M e SH2M são facilmente implementados usando a mesma estrutura de dados empregada nos métodos de elementos finitos de Galerkin contínuo, permitindo diferentes graus de interpolação polinomial para as variáveis locais e para o multiplicador favorecendo uma maior flexibilidade e uma maior precisão. Resultados numéricos mostraram taxas ótimas de convergência para as variáveis $\mathbf{u}_{h}$ e $p_{h}$ e para os multiplicadores $\boldsymbol{\lambda}_{\boldsymbol{u}_{\boldsymbol{h}}}$ e $\lambda_{p_{h}}$ inclusive para espaços polinomiais lagrangianos de mesma ordem com elementos triangulares e quadrilaterais. 


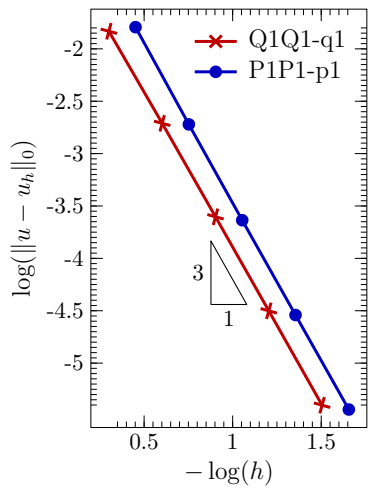

(a) $\mathbf{u}_{h}$

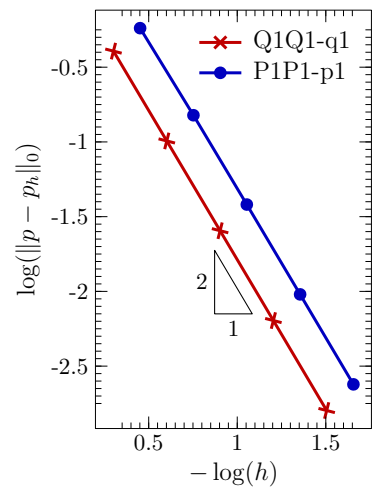

(b) $p_{h}$

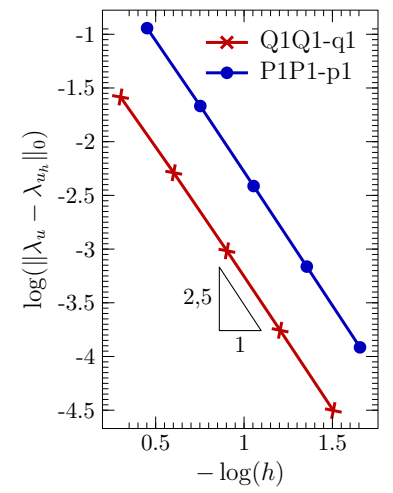

(c) $\boldsymbol{\lambda}_{u_{h}}$

Figura 5: Estudo de convergência $h$ das aproximações $\mathbf{u}_{h}, p_{h}$ e $\boldsymbol{\lambda}_{\boldsymbol{u}_{\boldsymbol{h}}}$ do método SH2M na norma $L^{2}(\Omega)$ comparando a precisão de elementos triangulares com elementos quadrilaterais utilizando interpolações quadráticas para a velocidade e multiplicador e linear para a pressão.

\section{Referências}

[1] D. N. Arnold and F. Brezzi. Mixed and nonconforming finite element methods: implementation, postprocessing and error estimates. Math. Model. Numer. Anal., 19:7-32, 1985.

[2] A. Baker, G., N. Jureidini, W., and A. Karakashian, O. Piecewise solenoidal vector fields and the stokes problem. SIAM J. Numer. Anal., 27(6):1466-1485, 1990.

[3] F. Brezzi and M. Fortin. Mixed and Hybrid Finite Element Methods. Springer-Verlag, 1991.

[4] B. Cockburn and J. Gopalakrishnan. New hybridization techniques. GAMM-Mitt., 2:154$183,2005$.

[5] B. Cockburn, J. Gopalakrishnan, and R. Lazarov. Unified hybridization of discontinuous galerkin, mixed, and continuous galerkin methods for second order elliptic problems. 47(2):1319-1365, 2009.

[6] B. Cockburn, G. Kanschat, D. Schötzau, and C. Schwab. Local discontinuous Galerkin methods for the Stokes system. SIAM J. Numer. Anal., 40(1):319-343, 2002.

[7] B. Cockburn, N. C. Nguyen, and J. Peraire. A comparison of hdg methods for stokes flow. J. Sci. Comput., 45:215-237, 2010.

[8] H. Egger and C. Waluga. hp analysis of a hybrid DG method for Stokes flow. IMA Journal of Numerical Analysis, 33:687-721, 2013.

[9] B. Fraejis de Veubeke. Displacement and equilibrium models in the finite element method, chapter 9, pages 145-197. John Wiley \& Sons, 1965.

[10] I. Igreja, C. O. Faria, and A. F. D. Loula. Hybridized mixed finite element method for the Stokes problem. Iberian Latin American Congress on Computational Methods in Engineering (CILAMCE), 2013, Pirenópolis. Anais Proceedings, 2013.

[11] P. A. Raviart and J. M. Thomas. Primal hybrid finite element methods for 2nd order elliptic equations. Math. Comp., 31(138):391 - 413, 1977.

[12] D. Schötzau, C. Schwab, and A. Toselli. Mixed hp-DGFEM for incompressible flows III: Pressure stabilization. Research Report, (2002-25), 2002.

[13] D. Schötzau, C. Schwab, and A. Toselli. Mixed hp-DGFEM for incompressible flows. SIAM J. Numer. Anal., 40:2171-2194, 2003. 